\title{
Factors related to household food insecurity in the Republic of Korea
}

\author{
Kirang Kim ${ }^{1,2, *}$, Mi Kyung Kim², Young-Jeon Shin ${ }^{2}$ and Sang Sun Lee ${ }^{1}$ \\ 'Department of Food and Nutrition, Hanyang University, Seoul 133-791, Republic of Korea: \\ ${ }^{2}$ Department of Preventive Medicine, College of Medicine, Hanyang University, Seoul, Republic of Korea
}

Submitted 6 May 2010: Accepted 7 December 2010: First published online 7 February 2011

\begin{abstract}
Objective: To examine the prevalence of food insecurity and to identify factors that contribute to it in the Republic of Korea.

Design: A cross-sectional study.

Setting: Data were selected from a secondary data set, the third Korean Welfare Panel Study. Household food insecurity was measured with a six-item Korean version of the US Household Food Security Survey Module. The differences in proportions or means of household characteristics, householder's characteristics, economic status and social benefits by food insecurity status were tested with the $\chi^{2}$ or $t$ test. The independent associations of food insecurity with each characteristic were assessed with multivariate logistic regression analysis.

Subjects: The sample size consisted of 6238 households.

Results: The prevalence of food insecurity was 5.3\% among all households and $25 \cdot 7 \%$ among low-income households. Risk factors that were associated with a higher risk of food insecurity included living alone, unemployment, no job, low household income and living in a leased or rented home. For low-income households, living in a leased or rented home increased the risk of food insecurity. Among food-insecure households, $26 \cdot 1 \%$ of the full sample of households and $34.3 \%$ of low-income households were participating in food assistance programmes.

Conclusions: Food insecurity among the Korean population was related to household type, income, job status and housing. Food assistance programmes were not enough to completely alleviate food insecurity.
\end{abstract}

\author{
Keywords \\ Food insecurity \\ Low-income household \\ Food assistance programmes
}

Food insecurity can be defined as the lack of availability of nutritionally adequate and safe foods or the lack of ability to acquire acceptable foods in socially acceptable ways $^{(1)}$. Food insecurity has been recognized as more than just a concern of underdeveloped or developing countries, as research has shown that even affluent Western nations share this problem ${ }^{(2)}$. Many countries have made efforts to define and measure the extent of these problems. On the basis of these efforts, research led to the development of methodologically sophisticated and empirically grounded measurement scales for food insecurity ${ }^{(1,3-6)}$. The experiential food insecurity measures have the advantage of incorporating as essential elements the perceptions of food insecurity by the people most affected, so that they are more direct measures of food insecurity than other proxy measures ${ }^{(1,4,6)}$. Currently, the measures have been extensively tested and proven robust in many countries ${ }^{(7-10)}$.

Several studies have examined the risk factors and consequences of food insecurity ${ }^{(1,3,11-13)}$. In particular, limited food accessibility, which is known to be affected by physical, social and economic resources, has been a major concern among members of modern society ${ }^{(14-18)}$. Many studies have examined the association between food insecurity and factors related to food acquisition, including social and demographic characteristics and financial resources ${ }^{(3,19-24)}$. The effects of these factors on food insecurity have been varied across a number of countries, regions and specific populations ${ }^{(10,21,23,25-29)}$. Understanding the factors related to the problems of food insecurity is crucial for addressing public nutritional policies and programmes, which provide nutrition and health services to populations with limited resources.

Recently, food insecurity has been recognized as an issue in Korean society and, as such, several studies have examined the nature of food insecurity in the Republic of Korea ${ }^{(30-33)}$. However, as these studies examined different populations and used a variety of different assessment measures, the prevalence of food insecurity has been estimated to range from $7 \cdot 6 \%$ to $62 \cdot 7 \%$. Recently, the 
nationwide prevalence of food insecurity has been evaluated using the six-item short form of the Korean Household Food Security Survey Module (KHFSS) from the Korean Welfare Panel Study (KoWePS) ${ }^{(34)}$. The purpose of the present study was to examine the prevalence of food insecurity and the factors that contribute to food insecurity in Korea.

\section{Methods}

\section{Study population and data collection}

Data were taken from KoWePS, which was conducted by the Korean Institute of Social and Health Affairs in conjunction with the Social Welfare Research Institute of Seoul National University. That study was designed to obtain nationally representative information on household financial status, housing, pension funds, employment histories, use of welfare services, health conditions and more, since the year 2006. The panel sample consisted of 14469 individuals from a national probability sample of 7072 households. The sampling frame was based on the Survey of Least Living Expenditures including 30000 households, which were selected by a two-stage stratified cluster sampling from 2005 census data. With the sampling design of a stratified systematic two-phase sampling, the panel sample was selected from the Survey of Least Living Expenditures on the basis of income levels, such that 3500 of the households sampled were low-income households under $60 \%$ of median income. The sample distribution according to income included $3789(53 \cdot 6 \%)$ participants over $60 \%$ of median income and $3283(46 \cdot 4 \%)$ participants under $60 \%$ of that. The data for the present study were taken from the third wave of the KoWePS (2008) because information on food insecurity has been recorded since 2008. Among the 6314 households surveyed in the third wave, households with missing values for food insecurity and income were excluded. The final sample for the analysis included 6238 households. Since no personal identifiers were used, the present study was exempt from human subjects review.

\section{Dependent variables}

Food insecurity was measured with the six-item short form of the KHFSS (Table 1). The KHFSS is an eighteen-item food insecurity index, which was previously developed and validated among adults aged $\geq 40$ years living in rural communities and among children participating in food assistance programmes ${ }^{(30)}$. This scale is based on the eighteen-item US Household Food Security Survey Module. According to the results of the previous study, when the six-item scale was compared with the eighteen-item scale, the classification power of the six-item scale was weaker for households with children than for households without children ${ }^{30)}$, which is consistent with the results of other studies $^{(7,35)}$. In the present study, the six-item questionnaire
Table 1 English reverse translation of the six-item food insecurity questionnaire from the Korea Household Food Security Survey Module*

\section{Instructions:}

There are several statements that people have made about their access to food. For these statements, please check whether the statement describes the level of access to food in your household during the last year (i.e. during the year 2007). Are there children under the age of 18 years or high-school students in your household? $\quad \square$ yes $\square$ no

1. In the year 2007 , because of economic difficulties, I did not have enough money to buy food even when I was out of food.

2. In the year 2007, because of economic difficulties, I could not afford to eat balanced meals (in sufficient amounts of various foods) because there was not enough money to buy food.

3 . In the year 2007, because of economic difficulties, have any adults in your household decreased the amount of meals or skipped meals because there was not enough money to buy food?

(a) (If you answered 'yes' to number 3) How often did this happen? $\square$ almost every month $\square$ some months, but not every month $\square$ only 1 or 2 months

4. In the year 2007, because of economic difficulties, have you eaten less than you felt you should because there was not enough money to buy food?

5. In the year 2007, because of economic difficulties, have you been unable to eat even when you were hungry because there was not enough money to buy food?

*Response options for items 1 and 2 were often, sometimes, never and do not know/refusal; response options for items 3,4 and 5 were yes, no and does not know/refusal.

had a Cronbach's $\alpha$ reliability coefficient of 0.85 and the point-biserial correlation coefficient for each item ranged from 0.49 for balanced meals to 0.69 for the decreased amount of meals or skipped meals.

In order to assess household food insecurity status, each item was assigned a score of 1 if the answer was affirmative (often/sometimes, yes, or almost every month/some months, but not every month) and 0 for all other responses. Households were classified into a food security group (additive total score $\leq 1$ ) and a food insecurity group (additive total score $\geq 2$ ) according to the household's additive total score. Households were defined as having low food security if the score was 2-4 and very low food security if the score was $\geq 5$. All questions were administered to participants by trained interviewers.

\section{Independent variables}

Factors related to food insecurity were examined across four areas: (i) household characteristics; (ii) householder's characteristics; (iii) factors related to economic status; and (iv) social benefits. The household characteristics included household type (two-adult headed, female head with no spouse, male head with no spouse, youth head, living alone), households with children of age $<18$ years, households with elders aged $>65$ years, elder-only households, households with disabled persons and family size.

Householder's characteristics included sex, age $(\geq 65$ years), education level (middle school or less ( $\leq 9$ years of schooling), high school (10-12 years of schooling), college or higher ( $\geq 13$ years of schooling)) and job status 
(none, unemployed, manual, non-manual). Non-manual jobs included managers, professionals, technicians and clerks, whereas manual jobs included service and sales workers, agricultural and fishery workers, craft and related trade workers, plant and machine operators and assemblers and unskilled workers.

Factors related to household economic status included household income, monthly food expenditure, the ratio of food to total expenditures and housing type. Household income was categorized into three groups according to the poverty index ratio (household income/national poverty line $\times 100$ ): $\leq 100 \%$ (poor households), 100-120\% (near poor households) and $>120 \%$ (middle- and higher-income households). Housing type was categorized into four groups, including one's own house, leased house, rented house and others. In a leased house, the tenant pays a large deposit at the time of signing the housing contract without paying monthly rent. In a rented house, the tenant pays a small deposit at the time of contract and also pays monthly rent. The other category included cases of living in a relative's home without paying rent.

Social benefit variables included the national basic living security and food assistance programmes. The beneficiary of national basic living security was defined as a household that has received cost of living, housing, medical, educational, childbirth, funeral and self-support benefits. Participation in food assistance programmes was defined as a household that has received free meal services or home-delivered meals during the last year.

\section{Statistical analysis}

The KoWePS includes post-stratification weight using 2005 census data, weight for primary sampling units and weight for an intentional over-sampling of low-income households ${ }^{(36)}$. Thus, all results were estimated using the sample weight. In the analysis of household income and food expenditures, household equivalence scales were applied to adjust for household size. The proportion or mean values of the factors by food insecurity status and the differences between food security and food insecurity were tested with $\chi^{2}$ tests for categorical variables and by $t$ tests for continuous variables. In order to examine the effects of factors on food insecurity, OR was estimated using the multivariate logistic regression analysis. The multivariate logistic regression analysis was used to select the best contributors among household characteristics, householder's characteristics, household economic status and participation in food assistance programmes. All analyses were conducted using the SAS statistical software package version $9 \cdot 1$ (SAS Institute Inc., Cary, NC, USA).

\section{Results}

The overall prevalence of household food insecurity is shown in Fig. 1. The prevalence of food insecurity was

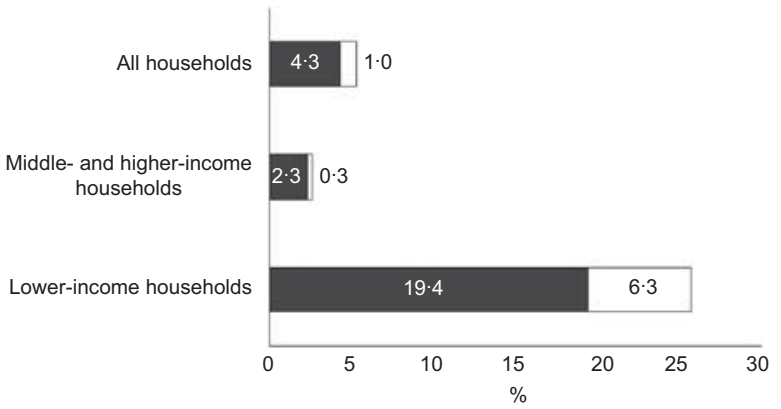

Fig. 1 The prevalence of household food insecurity ( $\square$, low food security; $\square$, very low food security) by household income level, Republic of Korea, 2007. Middle- and higher-income households represent households with a poverty index ratio (household income/national poverty line $\times 100$ ) $>120 \%$. The lower-income households represent households with a poverty index ratio $\leq 120 \%$

$5 \cdot 3 \%$. For low-income households (defined as households with a poverty index ratio $\leq 120 \%$ ), the prevalence of food insecurity was $25 \cdot 7 \%$, and, of these, $6 \cdot 3 \%$ had a very low food security status.

Table 2 describes household characteristics, householder's characteristics, economic status and social benefits by food insecurity status. Compared with food-secure households, food-insecure households were more likely to be headed by a single woman or man, to be single-person households (especially elder-only households) or to be households with elders or disabled persons. The proportion of households with children was higher among foodsecure households (42.9\%) than among food-insecure households $(31 \%)$, and the mean family size was larger among food-secure households $(2 \cdot 95 v .2 \cdot 17)$. With respect to householder's characteristics, food-insecure households had a relatively higher proportion of female $(40 \cdot 8 \% v$. $17 \cdot 5 \%)$ and elders as heads of the household (40.5\% v. $22.9 \%$ ) than did food-secure households. The householder's educational level was lower and the proportion of householders with an unstable job status (none or unemployment) was higher among food-insecure households than among food-secure households. Lower household income was related to a higher risk of food insecurity. About half of the food-insecure households were lowincome households (poverty index ratio $\leq 120 \%$ ), whereas most food-secure households had a poverty index ratio $>120 \%$. Monthly food expenditures among food-insecure households were lower compared with food-secure households. About half of the food-insecure households paid monthly rent, whereas $15.9 \%$ of food-secure households paid monthly rent. Of the food-insecure households, $36.5 \%$ received public assistance benefits and 26.1\% participated in food assistance programmes. Among lowincome households, the results were generally similar to those described above, with several exceptions. Specifically, the proportion of households with children did not differ between food-secure and food-insecure households, and the proportion of households with elders or 
Table 2 Characteristics of household by food insecurity status, Republic of Korea, 2007

\begin{tabular}{|c|c|c|c|c|c|c|c|c|}
\hline \multirow[b]{3}{*}{ Category } & \multicolumn{4}{|c|}{ All households } & \multicolumn{4}{|c|}{ Low-income households } \\
\hline & \multirow{2}{*}{$\frac{\text { Total }}{\%}$} & \multirow{2}{*}{$\frac{\begin{array}{c}\text { Food } \\
\text { security }\end{array}}{\%}$} & \multirow{2}{*}{$\frac{\begin{array}{c}\text { Food } \\
\text { insecurity }\end{array}}{\%}$} & \multirow[b]{2}{*}{$P$ value } & \multirow{2}{*}{$\frac{\text { Total }}{\%}$} & \multirow{2}{*}{$\frac{\begin{array}{c}\text { Food } \\
\text { security }\end{array}}{\%}$} & \multirow{2}{*}{$\frac{\begin{array}{c}\text { Food } \\
\text { insecurity }\end{array}}{\%}$} & \multirow[b]{2}{*}{$P$ value } \\
\hline & & & & & & & & \\
\hline \multicolumn{9}{|l|}{ Household characteristics } \\
\hline \multicolumn{9}{|l|}{ Household type } \\
\hline Two-adult headed & $81 \cdot 1$ & $82 \cdot 7$ & $53 \cdot 3$ & $<0.001$ & $51 \cdot 9$ & $54 \cdot 1$ & $45 \cdot 6$ & $<0.001$ \\
\hline Female head, no spouse & $1 \cdot 3$ & $1 \cdot 2$ & $2 \cdot 9$ & & $4 \cdot 3$ & $4 \cdot 3$ & $4 \cdot 3$ & \\
\hline Male head, no spouse & 0.5 & 0.5 & $1 \cdot 5$ & & 0.8 & $0 \cdot 8$ & 0.9 & \\
\hline Youth head & $0 \cdot 6$ & 0.5 & $2 \cdot 0$ & & $2 \cdot 5$ & $2 \cdot 4$ & $2 \cdot 7$ & \\
\hline Living alone & $16 \cdot 5$ & $15 \cdot 2$ & $40 \cdot 3$ & & $40 \cdot 5$ & $38 \cdot 4$ & $46 \cdot 5$ & \\
\hline Household with children aged $<18$ years & $42 \cdot 2$ & $42 \cdot 9$ & $31 \cdot 0$ & $<0.001$ & $26 \cdot 4$ & $26 \cdot 4$ & $26 \cdot 3$ & 0.07 \\
\hline Household with elders aged $\geq 65$ years & $30 \cdot 7$ & $29 \cdot 7$ & $47 \cdot 0$ & $<0.001$ & $62 \cdot 3$ & $64 \cdot 4$ & $56 \cdot 2$ & $<0.001$ \\
\hline Elder-only household & $8 \cdot 2$ & $7 \cdot 1$ & $26 \cdot 1$ & $<0.001$ & $31 \cdot 3$ & $30 \cdot 3$ & $34 \cdot 0$ & $<0.001$ \\
\hline Household with disabled persons & $15 \cdot 7$ & $15 \cdot 2$ & $25 \cdot 8$ & $<0.001$ & $31 \cdot 9$ & $32 \cdot 5$ & $30 \cdot 2$ & $<0.001$ \\
\hline Mean & $2 \cdot 92$ & $2 \cdot 95$ & $2 \cdot 17$ & 0.03 & $2 \cdot 13$ & $2 \cdot 15$ & $2 \cdot 05$ & $0 \cdot 18$ \\
\hline SE & 0.02 & 0.02 & 0.06 & & 0.04 & 0.04 & $0 \cdot 07$ & \\
\hline \multicolumn{9}{|l|}{ Householder's characteristics } \\
\hline Female & $18 \cdot 7$ & $17 \cdot 5$ & $40 \cdot 8$ & $<0.001$ & $50 \cdot 9$ & $50 \cdot 5$ & $52 \cdot 1$ & $<0.001$ \\
\hline$\geq 65$ years old & $23 \cdot 9$ & $22 \cdot 9$ & $40 \cdot 5$ & $<0.001$ & $55 \cdot 1$ & $56 \cdot 7$ & $50 \cdot 5$ & $<0.001$ \\
\hline \multicolumn{9}{|l|}{ Education level } \\
\hline College or higher & $32 \cdot 9$ & $34 \cdot 1$ & $10 \cdot 6$ & $<0.001$ & $6 \cdot 7$ & $6 \cdot 6$ & $7 \cdot 0$ & $<0.001$ \\
\hline High school & $33 \cdot 6$ & $34 \cdot 0$ & $26 \cdot 4$ & & $20 \cdot 3$ & $19 \cdot 8$ & $21 \cdot 8$ & \\
\hline Middle school or lower & $33 \cdot 6$ & $31 \cdot 9$ & $63 \cdot 0$ & & $73 \cdot 0$ & $73 \cdot 6$ & $71 \cdot 2$ & \\
\hline \multicolumn{9}{|l|}{ Job } \\
\hline Non-manual & $23 \cdot 9$ & $25 \cdot 2$ & $2 \cdot 3$ & $<0.001$ & 1.9 & $2 \cdot 1$ & $1 \cdot 2$ & $<0.001$ \\
\hline Manual & $50 \cdot 3$ & $50 \cdot 8$ & $41 \cdot 4$ & & $30 \cdot 3$ & $33 \cdot 3$ & $21 \cdot 7$ & \\
\hline Unemployment & $2 \cdot 8$ & $2 \cdot 6$ & $6 \cdot 9$ & & 3.5 & $2 \cdot 2$ & $7 \cdot 2$ & \\
\hline None & $22 \cdot 9$ & $21 \cdot 4$ & $49 \cdot 4$ & & $64 \cdot 3$ & $62 \cdot 4$ & $70 \cdot 0$ & \\
\hline \multirow{2}{*}{\multicolumn{9}{|c|}{$\begin{array}{l}\text { Factors related to economic status } \\
\text { Household income }(\mathrm{PIR})^{\star}\end{array}$}} \\
\hline & & & & & & & & \\
\hline$>120$ & $90 \cdot 1$ & $92 \cdot 4$ & $48 \cdot 5$ & & & & & \\
\hline $100-120$ & $1 \cdot 9$ & $1 \cdot 6$ & $8 \cdot 1$ & & & & & \\
\hline$\leq 100$ & $8 \cdot 0$ & $6 \cdot 0$ & $43 \cdot 4$ & $<0.001$ & & & & \\
\hline \multicolumn{9}{|l|}{ Monthly food expenditure ( $\$ 1000)$} \\
\hline Mean & $346 \cdot 0$ & $353 \cdot 0$ & $220 \cdot 0$ & $<0.001$ & $202 \cdot 0$ & $207 \cdot 0$ & $187 \cdot 0$ & $<0.001$ \\
\hline SE & 1.9 & 1.9 & $4 \cdot 2$ & & 0.5 & $2 \cdot 6$ & 0.4 & \\
\hline \multicolumn{9}{|l|}{ Percentage of food to total expenditure } \\
\hline Mean & $25 \cdot 6$ & $25 \cdot 3$ & $31 \cdot 1$ & $<0.001$ & $35 \cdot 3$ & $35 \cdot 9$ & $33 \cdot 4$ & $<0.001$ \\
\hline SE & $0 \cdot 1$ & $0 \cdot 1$ & 0.5 & & 0.3 & 0.4 & 0.6 & \\
\hline \multicolumn{9}{|l|}{ Housing type } \\
\hline One's own house & $54 \cdot 3$ & $56 \cdot 5$ & $17 \cdot 3$ & $<0.001$ & $21 \cdot 5$ & $26 \cdot 0$ & $8 \cdot 5$ & $<0.001$ \\
\hline Leased houset & $18 \cdot 8$ & $18 \cdot 9$ & $17 \cdot 0$ & & $17 \cdot 4$ & $16 \cdot 8$ & $19 \cdot 2$ & \\
\hline Rented houseł & $18 \cdot 0$ & $15 \cdot 9$ & $54 \cdot 1$ & & $39 \cdot 2$ & $32 \cdot 6$ & $58 \cdot 1$ & \\
\hline Others $\S$ & 8.9 & $8 \cdot 7$ & $11 \cdot 6$ & & $21 \cdot 9$ & $24 \cdot 6$ & $14 \cdot 2$ & \\
\hline Social benefits & & & & & & & & \\
\hline Public assistancell & $7 \cdot 2$ & $5 \cdot 5$ & $36 \cdot 5$ & $<0.001$ & $60 \cdot 6$ & $59 \cdot 3$ & $64 \cdot 4$ & $<0.001$ \\
\hline Food assistance programmes & $9 \cdot 4$ & $8 \cdot 4$ & $26 \cdot 1$ & $<0.001$ & $32 \cdot 5$ & $31 \cdot 8$ & $34 \cdot 3$ & $<0.001$ \\
\hline
\end{tabular}

PIR, poverty index ratio.

${ }^{*} \mathrm{PIR}=$ household income/national poverty line $\times 100$.

tPaying a large deposit at the time of signing a housing contract without paying monthly rent.

fPaying a small deposit at the time of signing a housing contract while paying monthly rent.

$\S$ Living in a relative's house without paying rent.

IIThe national basic living security scheme providing cost of living, housing, medical, educational, childbirth, funeral and self-support benefits.

- Participation in free meal services or home-delivered meal services.

disabled persons was higher among food-secure households. The proportion of recipients of public assistance and participation in food assistance programmes among low-income food-insecure households were $64 \cdot 4 \%$ and $34 \cdot 3 \%$, respectively.

The effects of factors related to household food insecurity were examined using multivariate logistic regression models (Table 3). Model 1 included household and householder's characteristics, and model 2 included factors related to economic status in addition to the variables in model 1. In the full model (model 3), because receiving public assistance and participation in food assistance programmes were highly correlated, each of these factors was examined separately with all of the other variables in model 2. Among the household characteristics in model 1 , households headed by a single woman or man, 
Table 3 Factors related to household food insecurity, Republic of Korea, 2007

\begin{tabular}{|c|c|c|c|c|c|c|c|c|}
\hline & \multicolumn{6}{|c|}{ All households } & \multirow{2}{*}{\multicolumn{2}{|c|}{$\frac{\text { Low-income households }}{\text { Model } 3}$}} \\
\hline & \multicolumn{2}{|c|}{ Model 1} & \multicolumn{2}{|c|}{ Model 2} & \multicolumn{2}{|c|}{ Model 3} & & \\
\hline & OR & $95 \% \mathrm{Cl}$ & OR & $95 \% \mathrm{Cl}$ & OR & $95 \% \mathrm{Cl}$ & OR & $95 \% \mathrm{Cl}$ \\
\hline \multicolumn{9}{|l|}{ Household characteristics } \\
\hline \multicolumn{9}{|l|}{ Household type } \\
\hline Two-adult headed & $1 \cdot 00$ & Ref. & $1 \cdot 00$ & Ref. & $1 \cdot 00$ & Ref. & $1 \cdot 00$ & Ref. \\
\hline Female head, no spouse & $2 \cdot 27$ & $1 \cdot 05,4 \cdot 94$ & $1 \cdot 11$ & $0 \cdot 49,2 \cdot 53$ & $1 \cdot 03$ & $0 \cdot 45,2 \cdot 35$ & $0 \cdot 82$ & $0 \cdot 31,2 \cdot 18$ \\
\hline Male head, no spouse & $3 \cdot 69$ & $1 \cdot 31,10 \cdot 4$ & $2 \cdot 81$ & $0.92,8 \cdot 61$ & $2 \cdot 77$ & $0 \cdot 89,8 \cdot 54$ & $1 \cdot 11$ & $0 \cdot 15,7 \cdot 82$ \\
\hline Youth head & $1 \cdot 80$ & $0.69,4.69$ & $1 \cdot 28$ & $0.45,3.62$ & $1 \cdot 27$ & $0.44,3.63$ & $1 \cdot 49$ & $0 \cdot 43,5 \cdot 11$ \\
\hline Living alone & $3 \cdot 35$ & $2 \cdot 40,4 \cdot 67$ & $1 \cdot 84$ & $1 \cdot 29,2 \cdot 63$ & $1 \cdot 84$ & $1 \cdot 29,2 \cdot 62$ & $1 \cdot 34$ & $0 \cdot 80,2 \cdot 22$ \\
\hline \multicolumn{9}{|c|}{ Household with children aged $<18$ years } \\
\hline No & $1 \cdot 00$ & Ref. & $1 \cdot 00$ & Ref. & $1 \cdot 00$ & Ref. & $1 \cdot 00$ & Ref. \\
\hline Yes & 1.94 & $1 \cdot 39,2 \cdot 72$ & $1 \cdot 42$ & $0.99,2 \cdot 02$ & $1 \cdot 27$ & $0 \cdot 87,1 \cdot 87$ & $1 \cdot 14$ & $0.57,2 \cdot 27$ \\
\hline \multicolumn{9}{|c|}{ Household with elders aged $\geq 65$ years } \\
\hline No & $1 \cdot 00$ & Ref. & $1 \cdot 00$ & Ref. & $1 \cdot 00$ & Ref. & $1 \cdot 00$ & Ref. \\
\hline Yes & $1 \cdot 23$ & $0.75,2 \cdot 00$ & $1 \cdot 47$ & $0.87,2.49$ & $1 \cdot 45$ & $0.86,2 \cdot 45$ & $1 \cdot 14$ & $0.51,2.52$ \\
\hline \multicolumn{9}{|c|}{ Household with disabled persons } \\
\hline No & 1.00 & Ref. & 1.00 & Ref. & $1 \cdot 00$ & Ref. & $1 \cdot 00$ & Ref. \\
\hline Yes & $1 \cdot 62$ & $1 \cdot 22,2 \cdot 15$ & $1 \cdot 24$ & $0 \cdot 91,1 \cdot 70$ & $1 \cdot 22$ & $0.89,1.66$ & $0 \cdot 82$ & $0.54,1.24$ \\
\hline \multicolumn{9}{|l|}{ Householder's characteristics } \\
\hline \multicolumn{9}{|l|}{ Sex } \\
\hline Male & 1.00 & Ref. & $1 \cdot 00$ & Ref. & $1 \cdot 00$ & Ref. & $1 \cdot 00$ & Ref. \\
\hline Female & $1 \cdot 18$ & $0 \cdot 86,1 \cdot 61$ & 1.02 & $0 \cdot 74,1 \cdot 41$ & $1 \cdot 01$ & $0 \cdot 73,1 \cdot 39$ & 0.95 & $0.62,1.46$ \\
\hline \multicolumn{9}{|l|}{ Age (years) } \\
\hline$<65$ & $1 \cdot 00$ & Ref. & $1 \cdot 00$ & Ref. & $1 \cdot 00$ & Ref. & $1 \cdot 00$ & Ref. \\
\hline \multirow{2}{*}{\multicolumn{9}{|c|}{ Education level $^{*}$}} \\
\hline & & & & & & & & \\
\hline College or higher & 1.00 & Ref. & 1.00 & Ref. & $1 \cdot 00$ & Ref. & $1 \cdot 00$ & Ref. \\
\hline High school & $1 \cdot 43$ & $0 \cdot 93,2 \cdot 18$ & $1 \cdot 24$ & $0.80,1.93$ & $1 \cdot 24$ & $0.80,1.92$ & $0 \cdot 85$ & $0.38,1.89$ \\
\hline Middle school or lower & $2 \cdot 65$ & $1 \cdot 73,4.05$ & $2 \cdot 14$ & $1 \cdot 36,3 \cdot 35$ & $2 \cdot 10$ & $1 \cdot 34,3 \cdot 28$ & $1 \cdot 02$ & $0 \cdot 48,2 \cdot 17$ \\
\hline \multicolumn{9}{|l|}{ Job } \\
\hline Non-manual & $1 \cdot 00$ & Ref. & $1 \cdot 00$ & Ref. & $1 \cdot 00$ & Ref. & $1 \cdot 00$ & Ref. \\
\hline Manual & $5 \cdot 60$ & $2 \cdot 63,11 \cdot 9$ & $4 \cdot 82$ & $2 \cdot 25,10 \cdot 3$ & $4 \cdot 76$ & $2 \cdot 23,10 \cdot 2$ & $1 \cdot 04$ & $0.21,4.99$ \\
\hline Unemployment & $18 \cdot 9$ & $8 \cdot 06,44 \cdot 6$ & $12 \cdot 5$ & $5 \cdot 16,30 \cdot 4$ & $12 \cdot 3$ & $5 \cdot 08,29 \cdot 9$ & 3.98 & $0 \cdot 71,22 \cdot 3$ \\
\hline None & $11 \cdot 8$ & $5 \cdot 40,25 \cdot 9$ & $5 \cdot 80$ & $2 \cdot 58,13 \cdot 0$ & $5 \cdot 66$ & $2 \cdot 52,12 \cdot 7$ & $1 \cdot 78$ & $0.37,8.58$ \\
\hline \multicolumn{9}{|c|}{ Factors related to economic status } \\
\hline Household income (PIR, \%)† & & & & & & & 0.99 & $0.99,1.00$ \\
\hline$>120$ & & & $1 \cdot 00$ & Ref. & 1.00 & Ref. & & \\
\hline $100-120$ & & & $3 \cdot 89$ & $2 \cdot 35,6 \cdot 43$ & $3 \cdot 79$ & $2 \cdot 29,6 \cdot 29$ & & \\
\hline$\leq 100$ & & & $4 \cdot 56$ & $3 \cdot 29,6 \cdot 33$ & $4 \cdot 33$ & $3 \cdot 10,6 \cdot 05$ & & \\
\hline \multicolumn{9}{|l|}{ Housing type } \\
\hline One's own house & & & $1 \cdot 00$ & Ref. & $1 \cdot 00$ & Ref. & $1 \cdot 00$ & Ref. \\
\hline Leased house & & & $2 \cdot 48$ & $1 \cdot 67,3 \cdot 68$ & $2 \cdot 47$ & $1 \cdot 66,3 \cdot 66$ & $2 \cdot 84$ & $1 \cdot 46,5 \cdot 53$ \\
\hline Rented house§ & & & $6 \cdot 71$ & $4 \cdot 78,9 \cdot 43$ & $6 \cdot 50$ & $4 \cdot 61,9 \cdot 16$ & $4 \cdot 56$ & $2 \cdot 49,8 \cdot 34$ \\
\hline Others\| & & & $1 \cdot 71$ & $1 \cdot 08,2 \cdot 73$ & $1 \cdot 73$ & $1 \cdot 09,2 \cdot 76$ & $1 \cdot 36$ & $0 \cdot 69,2 \cdot 71$ \\
\hline \multicolumn{9}{|l|}{ Food assistance programmes } \\
\hline No & & & & & 1.00 & Ref. & $1 \cdot 00$ & Ref. \\
\hline Yes & & & & & $1 \cdot 30$ & $0.91,1.85$ & 0.90 & $0.56,1.44$ \\
\hline \multicolumn{9}{|l|}{ Recipients for public assistance ${ }^{\star \star}$} \\
\hline No & & & & & 1.00 & Ref. & $1 \cdot 00$ & Ref. \\
\hline Yes & & & & & $1 \cdot 21$ & $0 \cdot 83,1 \cdot 77+t$ & 0.92 & $0.61,1 \cdot 38$ \\
\hline
\end{tabular}

PIR, poverty index ratio; Ref., reference category.

Model 1 food insecurity = household characteristics + householder's characteristics.

Model 2 food insecurity = household characteristics + householder's characteristics + economic factors.

Model 3 food insecurity = household characteristics + householder's characteristics + economic factors + food assistance programmes

All statistical significance was tested at $P<0.05$ level.

${ }^{*} P$ for trend of risks across education level $\leq 0.0001$ for all households, NS for low-income households.

TPIR = household income/national poverty line $\times 100$.

łPaying a large deposit at the time of signing a housing contract without paying monthly rent.

§aying a small deposit at the time of signing a housing contract and paying monthly rent.

IILiving in a relative's house without paying rent.

- Participation in free meal services or home-delivered meal services.

**The national basic living security scheme providing cost of living, housing, medical, educational, childbirth, funeral and self-support benefits.

t+OR was estimated when recipients of public assistance were included in the full model, instead of participants in food assistance programmes.

were more likely to be food insecure than those without these types of household members (OR $=1.94,95 \% \mathrm{CI}$ $1 \cdot 39,2 \cdot 72$ for the households with children; $\mathrm{OR}=1 \cdot 62$, were more likely to be food insecure than other households. Households with children or disabled persons 
$95 \%$ CI $1 \cdot 22,2 \cdot 15$ for the households with disabled persons). With respect to householder's characteristics, lower educational level of the householder was associated with food insecurity. Households in which the householder had a manual job or was unemployed or jobless were more likely to be food insecure than those in which the householder had a non-manual job.

When factors related to economic status were examined with both household and householder's characteristics in model 2, it was determined that single-person households (living alone), householder's job status and all factors related to economic status were significantly related to the risk of food insecurity. Among these variables, factors reflecting unstable household economic resources, such as unemployment status or living in a rented home, showed strong associations with risk of food insecurity $(\mathrm{OR}=8 \cdot 75$, 95\% CI 3.58, 21.4 for unemployment status; OR $=7 \cdot 01$, 95\% CI 4.96, 9.92 for living in a rented home). When participation in food assistance programmes or public assistance was included in the model, the coefficients were low and not statistically significant $(\mathrm{OR}=1 \cdot 30,95 \% \mathrm{CI}$ $0.92,1.85$ for food assistance programmes; $\mathrm{OR}=1 \cdot 18$, $95 \%$ CI $0 \cdot 81,1.70$ for public assistance). Among lowincome households, in the full model, living in a leased or rented home was related to increased risk of food insecurity $(\mathrm{OR}=3 \cdot 06,95 \%$ CI $1 \cdot 57,5 \cdot 98$ for living in a leased house; $\mathrm{OR}=4 \cdot 62,95 \% \mathrm{CI} 2 \cdot 49,8 \cdot 55$ for living in a rented house). Participation in food assistance programmes and public assistance were not related to risk of food insecurity among low-income households.

\section{Discussion}

Given the globally increasing relevance of food insecurity, the present study examined the prevalence of food insecurity and risk factors associated with food insecurity among Korean households. The prevalence of food insecurity was $5 \cdot 3 \%$ in all of the households and $25 \cdot 7 \%$ in the low-income households examined (19.4\% for the low food security group and $6.3 \%$ for the very low food security group). The factors that were significantly associated with an increased risk of food insecurity included living alone, unstable job status/unemployment, low household income and living in a leased or rented home. For low-income households, living in a leased or rented home was significantly associated with a higher risk of food insecurity. Receiving public assistance and participating in food assistance programmes were not significantly related to food insecurity, even among low-income households.

Food insecurity was influenced by a number of household characteristics, as is consistent with the results of previous studies ${ }^{(9,10,20,21,25,37-40)}$. Households headed by a single woman or man, youth-headed households and single-person households had a higher risk of food insecurity, which is consistent with the results of other studies $^{(9,10,21,25,40)}$. However, these relationships became non-significant after adjusting for economic factors. In addition, for low-income households, these household characteristics were not significantly related to food insecurity. These findings imply that food insecurity of households with these demographic factors can be almost entirely traced to their poorer economic status. Interestingly, the findings related to households with children were different for the full sample of households and for the sample of low-income households. The proportion of households with children was lower among foodinsecure households than among food-secure households in the full sample, which was inconsistent with other studies $^{(10,40)}$, but these findings were reversed in the sample of low-income households. As such, this result was explained by the household's socio-economic status. The OR for households with children was 0.59 (95\% CI $0 \cdot 47,0 \cdot 76)$. However, after adjusting for other variables related to socio-economic status, households with children appeared to be at an increased risk of food insecurity. These findings represent a unique characteristic of low-income households in Korea. Currently, Korea has a very low fertility rate $(1 \cdot 19 / 1000 \text { persons in } 2008)^{(41)}$, and it is known that a household's economic status partly contributes to the number of children raised in a household $^{(9,42,43)}$. That is, because of the high cost of raising children, low-income households are less likely to have children. According to work by Kim, the relative poverty rate $(50 \%$ of median income) has increased from $16 \cdot 1 \%$ (2003) to $19 \cdot 7 \%$ (2006); however, that of households with children has decreased from $12 \cdot 6 \%$ to $11 \cdot 0 \%$.

The present study showed that unstable job status and housing type were risk factors for household food insecurity, even after adjusting for household income. In particular, housing type was significantly related to risk of food insecurity among low-income households. Rented houses in Korea have been shown to be associated with lower housing stability, amenities, quality and per capita living space, reflective of the household's socio-economic status ${ }^{(44,45)}$. Unemployment status or paying monthly rent may lead to a cut food expenditures when there are limited resources ${ }^{(46-48)}$. Kirkpatrick and Tarasuk $^{(46)}$ found that among low-income households, households that paid rent or mortgages had lower food expenditures than those without housing payments. These findings imply that a multifaceted intervention approach, such as appropriate labour market programmes, unemployment benefits and housing subsidies, as well as food assistance programmes, may be helpful in alleviating food insecurity by making family resources more stable and sustainable. The importance of stable and sustainable family resources is represented by the elderly population whose income is relatively more likely to be stable because of social security. Several studies showed that food-insecurity of elderly households was relatively lower than that of non-elderly households ${ }^{(20,49)}$. 
The present study also showed that, specifically for lowincome households, the proportion of households with elders was higher in food-insecure households than in food-secure households.

In situations in which poor people have to make choices among several basic necessitates with limited resources, food assistance programmes may be important in reducing food insecurity. Previous studies on the effects of participation in food assistance programmes have resulted in inconsistent findings ${ }^{(19-21,23,25,28,29,40)}$. In the present cross-sectional study, the association between food insecurity and participation in food assistance programmes was weak and not statistically significant. One reason could be that the research design was not able to control for self-selection of more food-needy households into the programme, leading to weakened effects of the programmes ${ }^{(19-21,29)}$. Several studies suggested that participation in programmes can be coincident with food insecurity rather than factors that contribute to or protect against food insecurity ${ }^{(21,50)}$. Another reason may be that the programmes did not completely meet the food needs of the participating household during the entire year as other studies showed, or households do not get assistance immediately upon becoming food insecure. In the present study, the prevalence of food insecurity among households participating in food assistance programmes was $29 \cdot 3 \%$ for the full sample, $45 \cdot 7 \%$ for elderly households and $13 \cdot 1 \%$ for households with children. Further studies providing better information on dynamic changes in food insecurity and programme participation will be needed to assess the impacts of participation in food assistance programmes on food insecurity.

The present study has several limitations. Because of the cross-sectional nature of the study, a causal association among the factors and food insecurity cannot be estimated. In addition, because the cross-sectional data lacked information on the pattern and period of programme participation, as well as the duration of food insecurity, unbiased effects of participation in programmes on food insecurity could not be assessed. With respect to the measurement of food insecurity, the sixitem index of the KHFSS used in the present study has not been validated among representative samples.

In conclusion, single-person households, low-income households, households whose heads are unemployed or jobless and households living in leased or rented homes were more likely to be food insecure. Specifically, living in leased or rented homes was strongly related to food insecurity among low-income households. Participation in food assistance programmes was not significantly related to food insecurity. These findings will be helpful in identifying segments of the population to be targeted for interventions. Furthermore, more active food assistance programmes with strategies to address the problems of unemployment and unstable housing are needed to alleviate food insecurity among the Korean population.

\section{Acknowledgements}

The present study received no specific grant from any funding agency in the public, commercial or not-forprofit sectors. None of the authors have any conflict of interest to declare. K.K., M.K.K. and Y.-J.S. conceived and designed the research; K.K. conducted all the statistical analyses and drafted the paper; M.K.K. and Y.-J.S. contributed to interpretation of the data and reviewed the manuscript; S.S.L. assisted in the study design and analyses. All authors have read and approved the final version of this manuscript.

\section{References}

1. Anderson SA (1990) Core indicators of nutritional state for difficult-to-sample populations. J Nutr 120, Suppl. 11, S1559-S1600.

2. Insel P, Turner RE \& Ross D (2004) World view of nutrition. In Nutrition, pp. 760-779 [P Insel, RE Turner and D Ross, editors]. London: Jones and Bartlett Publishers.

3. Campbell CC (1991) Food insecurity: a nutritional outcome or a predictor variable? J Nutr 121, 408-415.

4. Coates J, Webb P \& Houser R (2003) Measuring Food Insecurity: Going Beyond Indicators of Income and Anthropometry. Washington, DC: Food and Nutrition Technical Assistance Project, Academy for Educational Development.

5. Food and Agriculture Organization of the United Nations (2002) Measurement and Assessment of Food Deprivation and Undernutrition. International Scientific Symposium. Rome: FAO.

6. Habicht JP, Pelto G, Frongillo EA et al. (2004) Conceptualization and Instrumentation of Food Insecurity. Proceedings of the Workshop on the Measurement of Food Insecurity and Hunger. Washington, DC: National Academy Press.

7. Bickel G, Nord M, Price C et al. (2000) Measuring Food Security in the United States. Guide to Measuring Housebold Food Security, Revised 2000. Alexandria, VA: USDA/FNS.

8. Coates J, Frongillo EA, Rogers BL et al. (2006) Commonalities in the experience of household food insecurity across cultures: what are measures missing? J Nutr 136, issue 5, 1438S-1448S.

9. Office of Nutrition Policy and Promotion (2007) IncomeRelated Housebold Food Security in Canada. Report no. H164-42/2007E. Ottawa: Health Canada.

10. Temple JB (2008) Severe and moderate forms of food insecurity in Australia: are they distinguishable? Aust J Soc Issues 43, 649-668.

11. Food and Agriculture Organization of the United Nations (1996) Rome Declaration on World Food Security and World Food Summit Plan of Action. World Food Summit. Rome: FAO.

12. Riely F, Mock N, Cogill B et al. (1999) Food Security Indicators and Framework for Use in the Monitoring and Evaluation of Food Aid Programs. Report no. HRN-A-00-9800046-00. Washington, DC: FANTA/Academy for Educational Development/USAID.

13. US Agency for International Development (1992) Policy Determination 19: Definition of Food Security. Washington, DC: USAID.

14. Food and Agriculture Organization of the United Nations (1983) World Food Security: A Reappraisal of the Concepts and Approaches. Director General's Report. Rome: FAO.

15. Food and Agriculture Organization of the United Nations (2003) Trade Reforms and Food Security. Conceptualizing the Linkages. Rome: FAO. 
16. Baro M \& Deubel TF (2006) Persistent hunger: perspectives on vulnerability, famine and food security in Sub-Saharan Africa. Annu Rev Anthropol 35, 521-538.

17. Nord M, Hooper MD \& Hopwood HA (2008) Householdlevel income-related food insecurity is less prevalent in Canada than in the United States. J Hunger Environ Nutr 3 , $17-35$.

18. Nord M \& Hopwood HA (2008) A Comparison of Household Food Security in Canada and the United States, Economic Research Report no. 67. Washington, DC: USDA; available at www.ers.usda.gov/publications/err67

19. Alaimo K, Briefel RR, Frongillo EA et al. (1998) Food insufficiency exists in the United States: results from the third National Health and Nutrition Examination Survey (NHANES III). Am J Public Health 88, 419-426.

20. Nord M (2007) Characteristics of Low-Income Households with Very Low Food Security: An Analysis of the USDA GPRA Food Security Indicator. Economic Information Bulletin no. 25. Washington, DC: USDA.

21. Olson CM, Rauschenbach BS, Frongillo EA et al. (1997) Factors contributing to household food insecurity in a rural upstate New York country. Fam Econ Nutr Rev 10, 2-17.

22. Rose D (1999) Economic determinants and dietary consequences of food insecurity in the United States. J Nutr 129, issue 2, 517S-520S.

23. Rose D, Gundersen C \& Oliveira V (1998) Socio-Economic Determinants of Food Insecurity in the United States: Evidence from the SIPP and CSFII Datasets. Technical Bulletin no. 1869. Washington, DC: USDA.

24. NSW Center for Public Health Nutrition (2003) Food Security Options Paper: A Planning Framework and Menu of Options for Policy and Practice Interventions. Gladesville: NSW Department of Health.

25. Che J \& Chen J (2000) Food insecurity in Canadian households. Health Rep 12, 11-22.

26. Doocy S \& Burnham G (2006) Assessment of socioeconomic status in the context of food insecurity: implications for field research. World Health Popul 8, 32-42.

27. Moreira PA \& Padrao PD (2004) Educational and economic determinants of food intake in Portuguese adults: a crosssectional survey. BMC Public Health 4, 58.

28. Onianwa OO \& Wheelock GC (2006) An analysis of the determinants of food insecurity with severe hunger in selected southern states. South Rural Sociol 21, 80-96.

29. Wehler C, Weinreb LF, Huntington N et al. (2004) Risk and protective factors for adult and child hunger among lowincome housed and homeless female-headed families. Am J Public Health 94, 109-115.

30. Kim K \& Kim MK (2009) Development and validation of food security measure. Korean J Nutr 42, 374-385.

31. Oh SY \& Hong MJ (2003) Food insecurity is associated with dietary intake and body size of Korean children from low-income families in urban areas. Eur J Clin Nutr 57, 1598-1604.

32. Kim K, Hong SA \& Kim MK (2008) Nutritional status and food insufficiency of Korean population through the life-course by education level based on 2005 National Health and Nutrition Survey. Korean J Nutr 41, 667-681.

33. Shim JS, Oh K \& Nam CM (2007) Association of household food security with dietary intake - based on the Third (2005) Korean National Health and Nutrition Examination Survey (KNHANES III). Korean J Nutr 41, 174-183.

34. Kim K, Kim MK \& Shin YJ (2009) Household food insecurity and its characteristics in Korea. Health Soc Welfare Rev 29, 268-292.

35. Blumberg SJ, Bialostosky K, Hamilton WL et al. (1999) The effectiveness of a short form of the Household Food Security Scale. Am J Public Health 89, 1231-1234.

36. Kim MG, Yeo E \& Lee BJ (2006) Korea Welfare Panel Study Descriptive Report. Report no. 2006-23. Seoul: KIHASA/ Social Welfare Research Center of SNU.

37. Casey PH, Szeto K, Lensing S et al. (2001) Children in foodinsufficient, low-income families: prevalence, health, and nutrition status. Arch Pediatr Adolesc Med 155, 508-514.

38. Klesges LM, Pahor M, Shorr RI et al. (2001) Financial difficulty in acquiring food among elderly disabled women: results from the Women's Health and Aging Study. Am J Public Health 91, 68-75.

39. Tarasuk VS \& Beaton GH (1999) Women's dietary intakes in the context of household food insecurity. J Nutr 129, 672-679.

40. Nord M, Andrews M \& Carlson S (2008) Household Food Security in the United States in 2007. Washington, DC: USDA.

41. Ministry for Health Welfare and Family Affairs (2009) 2009 Yearbook of Health, Welfare and Family Statistics. Seoul: MHWFA.

42. Jeong JH, Hwang DS, Kum JH et al. (2005) A Study on the Working Poor in Korea. Report no. 2005-03. Seoul: Korea Labor Institute.

43. Lee HJ, Kim MG, Noe DM et al. (2006) The Structure of Poverty in Korea. Report no. 2006-10. Seoul: KIHSA/MHW.

44. Oh JH \& Yeo E (2009) Housing index on the basis of Korea Welfare Panel Study. Health Welfare Forum 12, 30-38.

45. Yoon J, Kim GY \& Park CG (2005) A Study on Housing Service Disparity among Regions and Classes (I): Development of Housing Service Indicators. Report no. 2005-40. Anyang: KRIHS.

46. Kirkpatrick S \& Tarasuk V (2003) The relationship between low income and household food expenditure patterns in Canada. Public Health Nutr 6, 589-597.

47. Levin CE, Ruel MT \& Morris SS (1999) Working women in an urban setting: traders, vendors and food security in Accra. World Dev 27, 1977-1991.

48. Kendig H (1984) Housing careers, life cycle and residential mobility: implications for the housing market. Urban Stud 21, 271-283.

49. Nord M (2002) Food security rates are high for elderly households. Food Rev 25, 19-24.

50. Kim K \& Frongillo EA (2009) Patterns of food insecurity and participation in food assistance programmes over time in the elderly. Public Health Nutr 12, 2113-2119. 